\title{
On cloud ice induced absorption and polarisation effects in microwave limb sounding
}

\author{
P. Eriksson ${ }^{1}$, B. Rydberg ${ }^{1}$, and S. A. Buehler ${ }^{2}$ \\ ${ }^{1}$ Department of Earth and Space Sciences, Chalmers University of Technology, Gothenburg, Sweden \\ ${ }^{2}$ Division of Space Technology, SRT, Luleå University of Technology, Kiruna, Sweden
}

Received: 8 February 2011 - Published in Atmos. Meas. Tech. Discuss.: 2 March 2011

Revised: 1 June 2011 - Accepted: 22 June 2011 - Published: 30 June 2011

\begin{abstract}
Microwave limb sounding in the presence of ice clouds was studied by detailed simulations, where clouds and other atmospheric variables varied in three dimensions and the full polarisation state was considered. Scattering particles were assumed to be horizontally aligned oblate spheroids with a size distribution parameterized in terms of temperature and ice water content. A general finding was that particle absorption is significant for limb sounding, which is in contrast to the down-looking case, where it is usually insignificant. Another general finding was that single scattering can be assumed for cloud optical paths below about 0.1 , which is thus an important threshold with respect to the complexity and accuracy of retrieval algorithms. The representation of particle sizes during the retrieval is also discussed. Concerning polarisation, specific findings were as follows: Firstly, no significant degree of circular polarisation was found for the considered particle type. Secondly, for the $\pm 45^{\circ}$ polarisation components, differences of up to $4 \mathrm{~K}$ in brightness temperature were found, but differences were much smaller when single scattering conditions applied. Thirdly, the vertically polarised component has the smallest cloud extinction. An important goal of the study was to derive recommendations for future limb sounding instruments, particularly concerning their polarisation setup. If ice water content is among the retrieval targets (and not just trace gas mixing ratios), then the simulations show that it should be best to observe any of the $\pm 45^{\circ}$ and circularly polarised components. These pairs of orthogonal components also make it easier to combine information measured from different positions and with different polarisations.
\end{abstract}

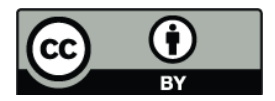

Correspondence to: P. Eriksson (patrick.eriksson@chalmers.se)

\section{Introduction}

The bulk of data provided so far by limb sounding sensors covers the stratosphere and the lower mesosphere, but recently both scientific objectives and the technical development have been moving the emphasis towards the upper troposphere (UT). The PREMIER mission proposal (ESA, 2008) is a good example for this change in scientific focus. Any satellite observation into the troposphere must deal with the impact of clouds, and this aspect is of special importance for limb sounding due to the long horizontal path lengths. In this respect, microwave techniques have an inherent advantage over infrared and optical techniques, because their sensitivity to clouds is significantly lower (e.g. Ekström et al., 2008).

In fact, a large fraction of microwave limb sounding data can be handled as "clear sky". The size of this fraction depends on the wavelengths used and the local atmospheric conditions. Nevertheless, cloud effects should be considered as this decreases the uncertainties of the gas species retrievals and increases the data yield. The treatment of clouds in typical microwave limb sounding gas inversion algorithms is highly simplified. They are mostly considered by a general continuum absorption term. Such a term is applied in the Odin-SMR (Murtagh et al., 2002) and Aura MLS (Waters et al., 2006) inversions, as described in Urban et al. (2005) and Livesey et al. (2006), respectively. The continuum absorption term is introduced primarily to account for uncertainties in absorption originating from non-local transitions and poorly understood physical mechanisms (see Rosenkranz, 1993). This term would also cover the impact of clouds if they could be treated as pure absorbers.

A more elaborated treatment of clouds, taking full account of scattering, is found in the methodology of Rydberg et al. (2009). Water vapour and cloud ice water content (IWC) are retrieved in parallel from Odin-SMR data, but only spectra

Published by Copernicus Publications on behalf of the European Geosciences Union. 
from single tangent altitudes are used and there is no obvious way to extend the approach to invert complete limb sounding sequences.

The interaction between microwave radiation and clouds is quite well studied for frequencies below $100 \mathrm{GHz}$ and ground-based or down-looking observation geometries (a review is given by Battaglia et al., 2006). The situation is quite different for frequencies above $150 \mathrm{GHz}$ and incidence angles near $90^{\circ}$, the conditions we study here. The basic justification of this study is that the development of more appropriate retrieval schemes requires a better understanding of how clouds affect microwave limb sounding radiances. The poorer knowledge for limb sounding could earlier be explained by a lack of relevant tools and input for the required simulations, but the situation has improved. Rigorous simulations of limb sounding measurements involving cloud scattering can now be performed by the ARTS software (Emde et al., 2004a; Davis et al., 2005a), and since 2006 the CloudSat $94 \mathrm{GHz}$ radar (Stephens et al., 2002) provides global information on cloud structures in a sufficiently detailed manner. ARTS and CloudSat are key components of our study.

For this study, the altitude region of interest is the UT and the focus is on wavelengths around $1 \mathrm{~mm}(300 \mathrm{GHz})$. The term " $\mathrm{mm}$ " is used below in favour of "microwaves" to make a clear distinction to the range below $100 \mathrm{GHz}$. The analysis is made primarily from the perspective of trace gas retrievals. The primary frequency range for such retrievals inside the UT is $150-700 \mathrm{GHz}$ (as indicated by the mm bands of Aura MLS). These observations can further provide information on IWC (Wu et al., 2005; Li et al., 2005; Eriksson et al., 2010) and this aspect is also considered.

Upper tropospheric clouds consist mainly of ice particles, and only this type of cloud is considered here. It has been established that scattering is in general the dominant process for ice clouds at mm wavelengths (e.g. Evans and Stephens, 1995b; Emde et al., 2004b). The relative importance of absorption is higher for small particles (Evans and Stephens, 1995a; Wu et al., 2005) and with a cold radiative background (Eriksson et al., 2008, and Sect. 3). These conditions are of special concern for limb sounding and ice particle absorption should be more prominent for this measurement technique, but this issue has not yet been studied in any detail.

Polarisation effects are of special importance for microwave sensors as these devices are polarisation sensitive. There are three main options for the response, in fact all covered by the last three $\mathrm{mm}$ limb sounders: Odin-SMR detects the $+45^{\circ}$ and $-45^{\circ}$ linearly polarised components (Eriksson et al., 2007), Aura MLS measures the horizontal (H) and vertical (V) linear components (Wu et al., 2006) and SMILES (Kikuchi et al., 2010) observes the left- and right-hand circular components (Y. Kasai, personal communication, 2010).

Even spherical particles cause some difference between the $\mathrm{V}$ and $\mathrm{H}$ components for $\mathrm{mm}$ limb sounding (Teichmann et al., 2006). Several studies have shown that this difference increases with the aspect ratio of the particles, on the condition that a preferred orientation exists. However, these studies have either been performed for a plane-parallel atmosphere (Czekala, 1998; Miao et al., 2003), down-looking geometry (Davis et al., 2007), for completely homogeneous (1-D) clouds (Davis et al., 2005b; Eriksson et al., 2007), or for just a single synthetic cloud (Emde et al., 2004a; Davis et al., 2005a,b). In addition, there exists no investigation of the other polarisation components with a limb sounding focus.

Our approach is to perform detailed three dimensional radiative transfer simulations, followed by careful analysis. The objectives of this work are:

1. Study the relative importance of ice particle absorption for mm limb sounding,

2. Calculate the complete polarisation state for a set of realistic scenarios, and,

3. Analyse the significance of the results for the retrievals.

The article is structures as follows: Sect. 2 presents some theoretical background and introduces tools and datasets used. Section 3 analyses the importance of particle absorption versus scattering. Section 4 presents and discusses the polarisation state in the simulations. Section 5 discusses cloud retrieval aspects. Finally, Sect. 6 contains a summary and the conclusions.

\section{Theory, tools and data}

\subsection{Radiative transfer}

The intensity and polarisation state of radiation are described by the Stokes vector, $\boldsymbol{I}$. The radiative transfer equation to be solved in the case of thermal emission and particle scattering is

$$
\begin{aligned}
\frac{\mathrm{d} \boldsymbol{I}(v, \boldsymbol{r}, \boldsymbol{n})}{\mathrm{d} s}= & -\mathbf{K}(v, \boldsymbol{r}, \boldsymbol{n}) \boldsymbol{I}(v, \boldsymbol{r}, \boldsymbol{n})+\boldsymbol{a}(v, \boldsymbol{r}, \boldsymbol{n}) B(v, \boldsymbol{r}) \\
& +\int_{4 \pi} \mathbf{Z}\left(v, \boldsymbol{r}, \boldsymbol{n}, \boldsymbol{n}^{\prime}\right) \boldsymbol{I}\left(v, \boldsymbol{r}, \boldsymbol{n}^{\prime}\right) \mathrm{d} \boldsymbol{n}^{\prime},
\end{aligned}
$$

where $v$ is the frequency, $\boldsymbol{r}$ is the atmospheric position, $\boldsymbol{n}$ is the propagation direction, $s$ is the distance along $\boldsymbol{n}, \mathbf{K}$ is the extinction matrix, $\boldsymbol{a}$ is the absorption vector, $B$ is the Planck function and $\mathbf{Z}$ is the scattering matrix. See Mishchenko et al. (2002) for details regarding the assumptions for this equation and definitions of the involved quantities. $\mathbf{Z}$ is frequently denoted as the phase matrix, but scattering matrix (e.g. Bohren and Huffman, 1998) is clearly a more descriptive name.

During the actual calculations the vector $\boldsymbol{I}$ holds radiance values. For the presentation, simulated radiances are converted to Planck brightness temperatures following the expressions in Eriksson et al. (2011). The standard nomenclature of denoting the four elements of the Stokes vector as 
$\boldsymbol{I}=[I, Q, U, V]^{T}$ is kept (Chandrasekhar, 1950), but they are here reported as

$$
\begin{aligned}
I & =\left(T_{B}^{v}+T_{B}^{h}\right) / 2, \\
Q & =T_{B}^{v}-T_{B}^{h}, \\
U & =T_{B}^{+45^{\circ}}-T_{B}^{-45^{\circ}}, \\
V & =T_{B}^{\mathrm{hc}}-T_{B}^{\mathrm{rhc}},
\end{aligned}
$$

where $T_{B}^{v}$ is the brightness temperature [K] for the vertically linearly polarised component, calibrated with respect to the single polarisation power of blackbody radiation. $T_{B}^{h}, T_{B}^{+45^{\circ}}$, $T_{B}^{-45^{\circ}}, T_{B}^{\mathrm{lhc}}$ and $T_{B}^{\mathrm{rhc}}$ are defined likewise, but consider the horizontal linear, $+45^{\circ}$ linear, $-45^{\circ}$ linear, left-hand circular, and right-hand circular component, respectively. The element $I$ could likewise be defined as the average of the other two pairs of orthogonal components $\left(T_{B}^{+45^{\circ}} / T_{B}^{-45^{\circ}}\right.$ and

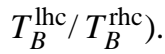

Conceptually, we study the impact of clouds by calculating the difference between each cloudy simulation and the corresponding clear-sky case, where all clouds are ignored. For the first Stokes component $I$, this difference is also what we are reporting:

$$
\Delta I=I-I_{C S}
$$

For the other three Stokes components, the clear-sky value is zero, as long as the tangent altitude is above the surface. We therefore simply report the $Q, U$, and $V$ values for the cloudy case.

Finally, note that the scattering of a particle is highly dependent on the ratio between its "characteristic size", $d$ and the wavelength, $\lambda$, normally reported as the size parameter, $x$ :

$x=\frac{\pi d}{\lambda}$.

That is, $x$ is the ratio between circumference and wavelength for spherical particles.

\subsection{Simulation software}

The simulations were performed by ARTS (Atmospheric Radiative Transfer Simulator), a freely available, open source, software package (Buehler et al., 2005). The second version of ARTS (Eriksson et al., 2011) includes two modules for solving Eq. (1). The Monte Carlo (MC) algorithm (Davis et al., 2005a) is used for this study. ARTS-MC allows simulation of cloud scattering in a three dimensional (3-D) atmosphere with arbitrary geoid and surface shapes, taking full account of polarisation effects. Gaseous absorption is calculated internally by ARTS, for efficiency reasons precalculated as a look-up table (Buehler et al., 2011). Particle optical (single scattering) properties were calculated with the T-matrix code by Mishchenko et al. (2002).

\subsection{Atmospheric scenarios}

It has been shown in several studies that the assumption of completely homogeneous cloud layers leads to a misrepresentation of cloud effects in limb observation geometry (Emde et al., 2004a; Davis et al., 2005a, 2007; Eriksson et al., 2007; Adams et al., 2008). We therefore used data on both horizontal and vertical cloud structure from CloudSat, a satellite-based cloud radar.

A methodology to make use of radar observations for simulating cloud effects in passive mm-wave data was developed by Rydberg et al. (2007), and later extended to 3-D in order to create a database for Odin-SMR upper tropospheric water retrievals (Rydberg et al., 2009). This study makes use of the atmospheric scenarios generated for the Odin-SMR database. In short, along-track cross-sections of radar back-scattering from CloudSat are transformed to 3-D fields using the algorithm by Venema et al. (2006). The 3D fields obtained are converted to fields of number densities using the particle size distribution (PSD) parametrisation by McFarquhar and Heymsfield (1997, below MH97). No retrievals are involved and the final data are fully consistent with the basic observations of CloudSat (see further Sect. 2.4). The database covers only tropical conditions (latitudes of $\pm 30^{\circ}$ ).

Rydberg et al. (2009) have shown that these atmospheric scenarios result in simulations that reproduce Odin-SMR observations in detail. This indicates that cloud structures above $10 \mathrm{~km}$ and over horizontal distances below $\sim 40 \mathrm{~km}$ (the footprint size of the satellite data considered) are reasonably represented. To what extent the 3-D distribution of temperature and gas species is correctly captured is not known. Particularly uncertain are the relationships between cloudy regions and the surrounding air, but in this context these aspects are secondary to the representation of clouds.

\subsection{Simulation details}

This work is part of a design study of the mm-wave limb sounder instrument for the PREMIER mission, and the frequency for the simulations was selected accordingly: $347.5 \mathrm{GHz}$. This is a frequency in-between transitions of $\mathrm{CO}$ and $\mathrm{HCN}$ (at 345.8 and $354.5 \mathrm{GHz}$, respectively), lacking local spectral features. The frequency $347.5 \mathrm{GHz}$ corresponds roughly to the lowest opacity with respect to gaseous absorption for the instrument and can be treated as a "window frequency".

The absorption for water, oxygen and nitrogen was taken from Rosenkranz (1998), Rosenkranz (1993), and Liebe et al. (1993), respectively. To this the absorption of roughly 70 transitions of other species was added. Figure 1 summarises the tropical average clear-sky radiative properties at $347.5 \mathrm{GHz}$. The opacity at this wavelength of $0.86 \mathrm{~mm}$ is roughly halfway between the one at $230 \mathrm{GHz}$ and the one at $501 / 645 \mathrm{GHz}$, the "window" frequencies of Aura MLS and 


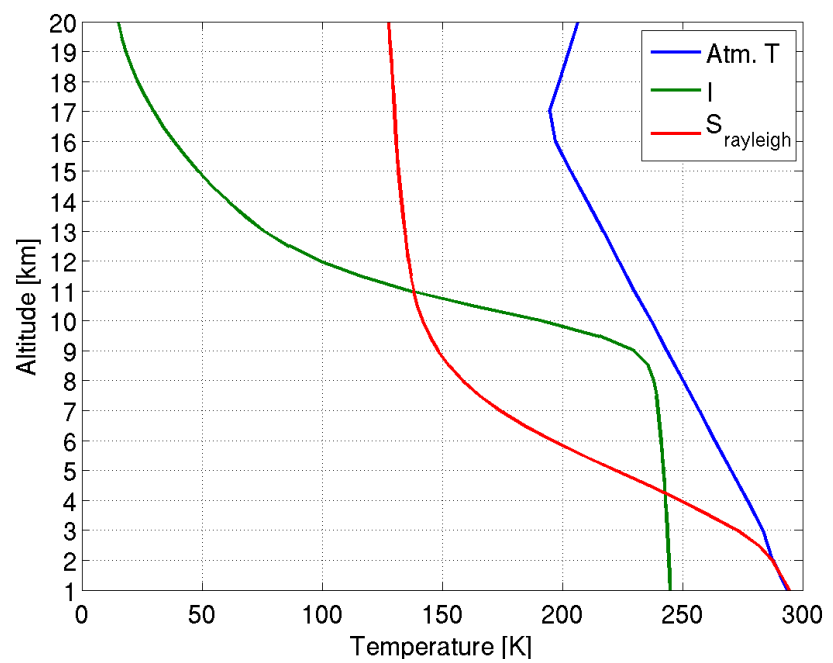

Fig. 1. Tropical mean atmospheric temperature as a function of altitude (blue), measured $I_{c S}$ is a function of tangent altitude (green), and the scattering source function $(S)$ as a function of altitude (red). The calculation of $S$ assumes single scattering conditions, Rayleigh conditions and that the zenith angle of $\boldsymbol{n}$ is $90^{\circ}$ (Eq. 10). The frequency is $347.5 \mathrm{GHz}$ and the data represent average tropical conditions (FASCODE).

Odin-SMR/SMILES, respectively. The figure also shows the scattering source term, which will be explained further down in Sect. 3.3.

The surface was assumed to have a spherical shape and to act radiatively as a blackbody. Monochromatic pencil beam simulations were performed for tangent altitudes of 4, 12, 14 and $16 \mathrm{~km}$, with refraction neglected. The basic properties of results for 14 and $16 \mathrm{~km}$ are the same, and only results for $14 \mathrm{~km}$ are shown.

No liquid cloud particles were included, only ice particles. The complete particle size distribution was represented by ten discrete sizes. In contrast to the original data by Rydberg et al. (2009), which assumed spherical cloud ice particles, the shape of the particles was assumed to be (solid) oblate spheroids, with the larger dimension in the horizontal plane. Two aspect ratios were considered: 1.2 and 2.0. The first value is based on an estimate by Davis et al. (2005b), who used simultaneous $\mathrm{V}$ and $\mathrm{H}$ Aura MLS $122 \mathrm{GHz}$ data. The second value is motivated by the fact that cirrus cloud particles are known to occasionally have very high aspect ratios, with orientation as assumed here (e.g. Okamoto et al., 2010).

Different approaches are possible when preparing particle populations with the same size distribution, but different aspect ratio. Our approach here was that for each of the ten size bins the particle number density field and the particle volume are identical for both aspect ratios. As a consequence, the IWC (in $\mathrm{gm}^{-3}$ ) is the same, independently of aspect ratio. Note that this approach is not maintaining a constant radar backscatter, and thus the perfect matching with the CloudSat observations (Sect. 2.3) is lost. However, in this case it was judged to be the best alternative for the interpretation of the results.

Finally, we emphasise that the performed radiative transfer simulations dealt with the full three dimensional spherical geometry and considered the full polarisation state.

\section{Absorption vs. scattering}

The focus in this section is on the first Stokes element $(I)$ and expressions considering just this element are used. This is denoted as "scalar radiative transfer". These simplified expressions are also better suited for explaining the results.

\subsection{Scalar radiative transfer}

If polarisation effects can be neglected, the radiative transfer over a short part of the propagation path, extending between points $i$ and $i+1$, can be approximated as

$I(i+1)=I(i) e^{-k l}+\left(1-e^{-k l}\right)[(1-\omega) B+\omega S]$,

where $I(i)$ is the intensity (for $\boldsymbol{n}$ ) at point $i, l$ is the distance along the path between $i$ and $i+1, k$ is the extinction coefficient (scalar equivalent to $\mathbf{K}$ ), $\omega$ is the single scattering albedo and $S$ is the source function for scattering. The last two quantities are defined as

$\omega=\frac{k-a}{k}=\frac{s}{k}$,

$S=\int_{4 \pi} p\left(\boldsymbol{n}, \boldsymbol{n}^{\prime}\right) I\left(\boldsymbol{n}^{\prime}\right) \mathrm{d} \boldsymbol{n}^{\prime}$,

where $a$ (scalar equivalent to $\boldsymbol{a}$ ) and $s$ are the absorption and scattering coefficients, respectively, and $p$ is the normalised scattering function. The normalisation is such that

$\int_{4 \pi} p\left(\boldsymbol{n}, \boldsymbol{n}^{\prime}\right) \mathrm{d} \boldsymbol{n}^{\prime}=1$.

Except for the normalisation, $p$ is the scalar equivalent to $\mathbf{Z}$.

\subsection{Extinction}

The extinction has two components, absorption and scattering out of the line-of-sight. The relative size of the two components is normally reported as the single scattering albedo (Eq. 9). Examples are shown in Fig. 2. The single scattering albedo for spherical particles depends on two quantities, the size parameter and the complex refractive index $\left(n=n^{\prime}+i n^{\prime \prime}\right)$. The real part of the refractive index of ice, $n^{\prime}$, is $\approx 1.8$ throughout the microwave region and the imaginary part, $n^{\prime \prime}$, increases smoothly from 0.001 around $80 \mathrm{GHz}$ to 0.01 around $700 \mathrm{GHz}$ (Warren and Brandt, 2008).

The data in Fig. 2 correspond to size parameters $x$ between 0.04 and 3.7, and thus either Rayleigh conditions $(x \ll 1)$ or Mie conditions $(x \approx 1)$ apply. For the Rayleigh regime, the 


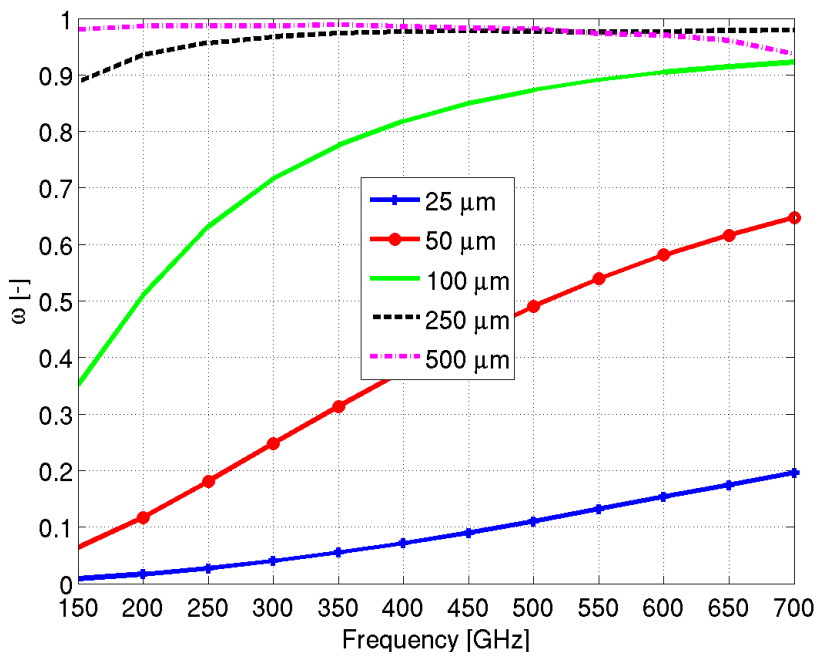

Fig. 2. Single scattering albedo $(\omega)$ for five different diameters of spherical ice particles.

absorption and scattering cross-sections are proportional to $d^{3} / \lambda$ and $d^{6} / \lambda^{4}$, respectively. These relationships explain in overall terms why the particle size is the main driver for $\omega$ (for the conditions spanned by the figure). Particles larger than about $250 \mu \mathrm{m}$ have an $\omega$ close to 1 throughout the $\mathrm{mm}$ range. For smaller particles, the increase of $\omega$ as a function of frequency is smaller than expected from the Rayleigh expressions, due to the counteracting change in $n^{\prime \prime}$.

To better consider real conditions, the mix of different particle sizes must be considered, i.e. the PSD. This has been done in Fig. 3 where the absorption and scattering coefficients have been summed up following the MH97 PSD (Sect. 2.3) and the bulk $\omega$ has been calculated. A more detailed analysis reveals that, for MH97, the contribution to the absorption term peaks for particles around $50 \mu \mathrm{m}$, while scattering peaks in the 150-300 $\mu \mathrm{m}$ size range (Wu et al., 2005; Eriksson et al., 2008). MH97 achieves a higher IWC primarily by increasing the number of particles larger than about $100 \mu \mathrm{m}$. Accordingly, $\omega$ is found to monotonically increase with IWC in Fig. 3.

\subsection{Source terms}

In principle, there is a single source to the measured radiance, thermal emission originating inside the atmosphere (cosmic background radiation, solar radiation, and surface emission give only very small contributions for the frequency and viewing geometry considered here). However, both with respect to physical and simulation aspects there is a large difference if the radiation is emitted directly or is scattered into the line-of-sight, and it therefore makes sense to also separate the source into two terms: emission and scattering.

The emission is directly linked to absorption as long as local thermodynamic equilibrium (LTE) applies, which is im-

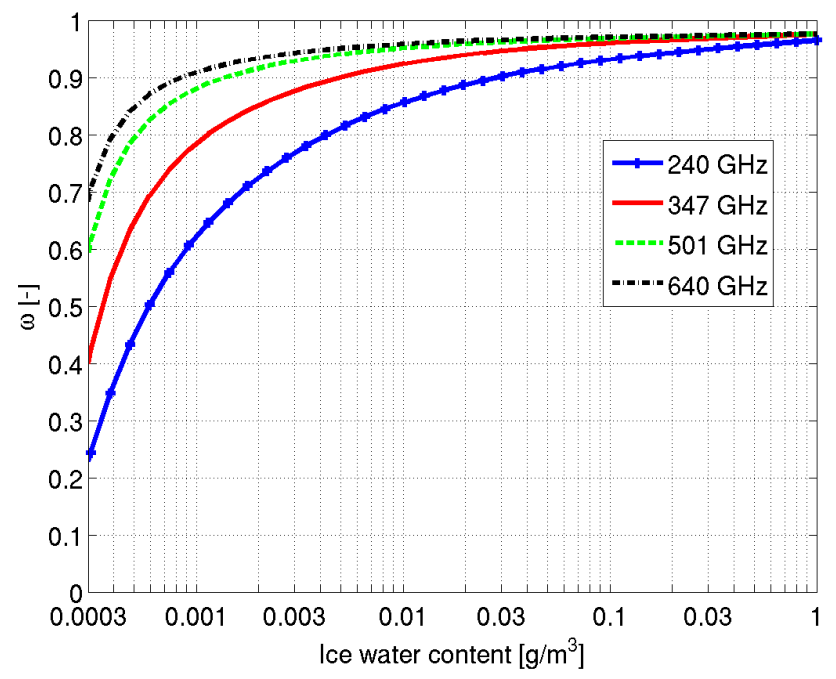

Fig. 3. Single scattering albedo $(\omega)$ for spherical ice particles as function of IWC for 4 different frequencies. Particle size distribution according to MH97 for $220 \mathrm{~K}$.

plied by Eqs. (1) and (8). The emission generated along a small path segment $\Delta l$ is $a B \Delta l$.

The scattering into the line-of-sight for the same segment is $s S \Delta l$. That is, both the emission and scattering source terms ( $a B$ and $s S$, respectively) are proportional to the corresponding extinction coefficient. (For vector radiative transfer it is, in general, not possible to express the scattering source term as the product of a scattering coefficient and a normalised scattering function, as for the scalar case here.)

As its absorption counterpart, the emission source function depends only on the local conditions. Expressed in brightness temperature, the Planck function is $B=T$. The scattering source function, $S$, behaves completely differently, it depends both on the scattering function $(p)$ and non-local conditions though the incoming radiation field (Eq. 11). Figure 1 exemplifies $S$ for the case of small particles and weak scattering.

\subsection{Discussion}

A straightforward test of the importance of ice particle absorption is to repeat some simulations with the scattering quantities set to zero. Results from such a test are shown in Fig. 4. The simulations were performed with vector radiative transfer, but the results can be understood in the scalar framework.

Let us first assume that scattering dominates totally, which is the general assumption that is challenged. In this case, Eq. (8) can be written as $(k=s)$

$\frac{\mathrm{d} I}{\mathrm{~d} l}=s(S-I)$. 
This equation shows that a fixed scattering coefficient $s$ gets its maximum impact when there is a large difference between $I$ and $S$. The case of $I \gg S$ is for longwave radiation only encountered when the observation direction is directly towards the sun and is not relevant here. The other extreme situation is $I=0$.

Equation 12 explains why $\Delta I$ in Fig. 4 for 4 and $14 \mathrm{~km}$ tangent altitude has opposite sign. For $14 \mathrm{~km}$, the clear-sky $I$ is about $50 \mathrm{~K}$, while $S$ is in the order of $130 \mathrm{~K}$ (Fig. 1). Thus, $S>I$ and scattering increases the measured intensity; the source term exceeds the extinction. The situation for $4 \mathrm{~km}$ is reversed. The extinction acts here on radiation originating from the lower troposphere and $I>S$ and the impact of clouds is to decrease the observed radiance.

This difference between "low" and "high" tangent altitudes is well-known (Emde et al., 2004b; Wu et al., 2005; Ekström et al., 2007). This study deals primarily with the "high" case. The 'low' case is basically parallel to the downlooking geometry considered for dedicated cloud ice sensors (Evans et al., 2002; Buehler et al., 2007). The transition from positive to negative change in $I$ due to cloud scattering depends on the position of the particles with respect to the tangent point, but occurs roughly at (the highest) altitude where the green and red lines in Fig. 1 cross.

Let us now consider the reverse case, that absorption dominates and scattering can be neglected. Then:

$\frac{\mathrm{d} I}{\mathrm{~d} l}=a(B-I)$.

Equations (12) and (13) follow the same pattern, but the fact that in general $B>S$ causes important differences. In the case of the $4 \mathrm{~km}$ tangent altitude, $I$ is just slightly larger than $B$; the absorbed part is replaced with emission of more or less the same power. This explains why $\Delta I_{\mathrm{abs}}$ is close to zero for the $4 \mathrm{~km}$ data in Fig. 4.

For $14 \mathrm{~km}$, we have a parallel situation to the purely scattering case. However, $B-I$ is roughly twice as large as $S-I$ (assuming the conditions of Fig. 1) and some absorption extinction gives a higher $\mathrm{d} I / \mathrm{d} l$ than scattering of same strength $(a=s)$. This was pointed out in Eriksson et al. (2008), but not explained in any detail.

Figure 4 shows this in practice, where on average $\Delta I_{\mathrm{abs}}$ is about $25 \%$ of $\Delta I$ although $\omega>0.8$ for most cases (Fig. 3). The varying distance to the 1-to-1 line is caused by multiple-scattering effects, and the fact that some cases have widespread clouds with low IWC, and then relatively low $\omega$, while others are more compact with high IWC and $\omega$.

If $I=S$ then scattering gives no net effect $(\mathrm{d} I / \mathrm{d} l=0)$. If $B>S$ for the same position there is a net contribution through the absorption coefficient. Hence, at positions with these conditions, the ice particles effectively act as $\omega=0$ independently of the actual $\omega$. This small example shows that the single scattering albedo $\omega$ only gives partial information on the relative importance of absorption and scattering for measurements of atmospheric emission. For "low" tangent

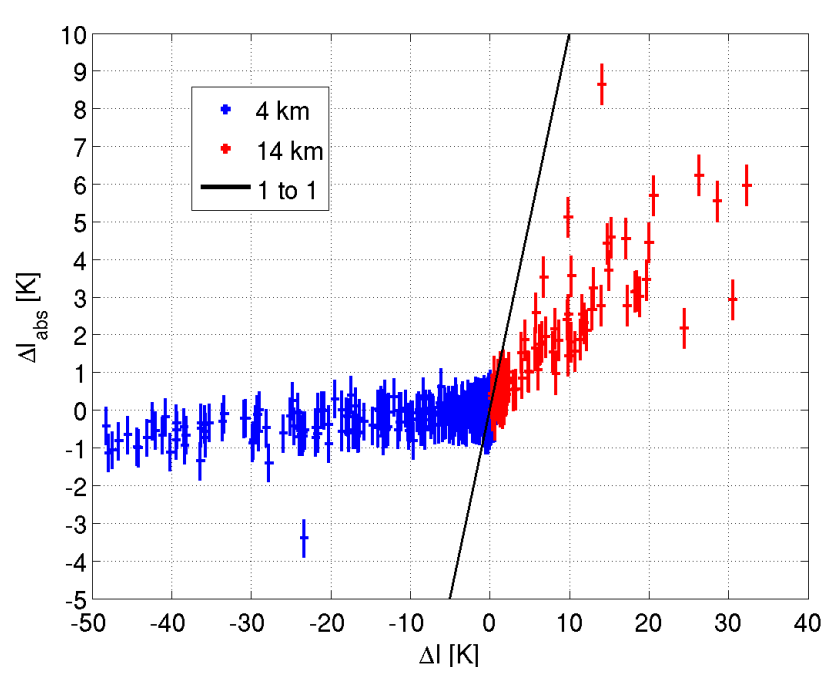

Fig. 4. The ice cloud induced change in intensity when treating the particles as purely absorbing $\left(\Delta I_{\mathrm{abs}}\right)$, as a function of $\Delta I$ for complete simulations. The $\Delta I_{\mathrm{abs}}$ case was achieved by setting both the scattering matrix and the scattering part of the extinction matrix to zero. The vertical lines show the uncertainty of the scattering calculations $( \pm 2 \sigma)$, originating from the Monte Carlo approach. Two tangent altitudes were considered, 4 (blue) and 14 (red) km. If scattering would have had a zero contribution, the results would have ended up around the 1 to 1 line (black). The simulations were performed for a frequency of $347.5 \mathrm{GHz}$ and three dimensional tropical atmospheric data (Sect. 2.3). The particles were assumed to be horizontally aligned oblate spheroids with an aspect ratio of 2 .

altitudes, scattering dominates even for small $\omega$. For "high" tangent altitudes, on the other hand, emission plays a larger role than expected, even for large $\omega$.

\section{Polarisation}

Figures 5 and 6 show the simulation results used in this section. The optical paths $\tau^{I}$ for the figures were calculated by propagating a unit Stokes vector $\left([1,0,0,0]^{T}\right)$ through the atmosphere considering only extinction, and taking $\tau^{I}=$ $-\log (I)$. The cloud optical paths $\tau_{\text {cloud }}^{I}$ were obtained by subtracting the $\tau_{\text {clear }}^{I}$ of the corresponding clear-sky calculations from the total optical paths $\tau^{I}$.

Figure 5 shows results for $14 \mathrm{~km}$ tangent altitude. For this tangent altitude the cloud induced change in intensity is strictly positive, in line with the "high" case discussed in Sect. 3.4. We will come back to this figure in later subsections.

Figure 6 shows results for $12 \mathrm{~km}$ tangent altitude. For this tangent altitude the cloud induced change in intensity can be positive or negative for the same cloud optical path $\tau_{\text {cloud }}^{I}$. As a help to understand this spread of $\Delta I$, the clear-sky optical path $\tau_{\text {clear }}^{I}$ is included in the figure by colour-coding the 

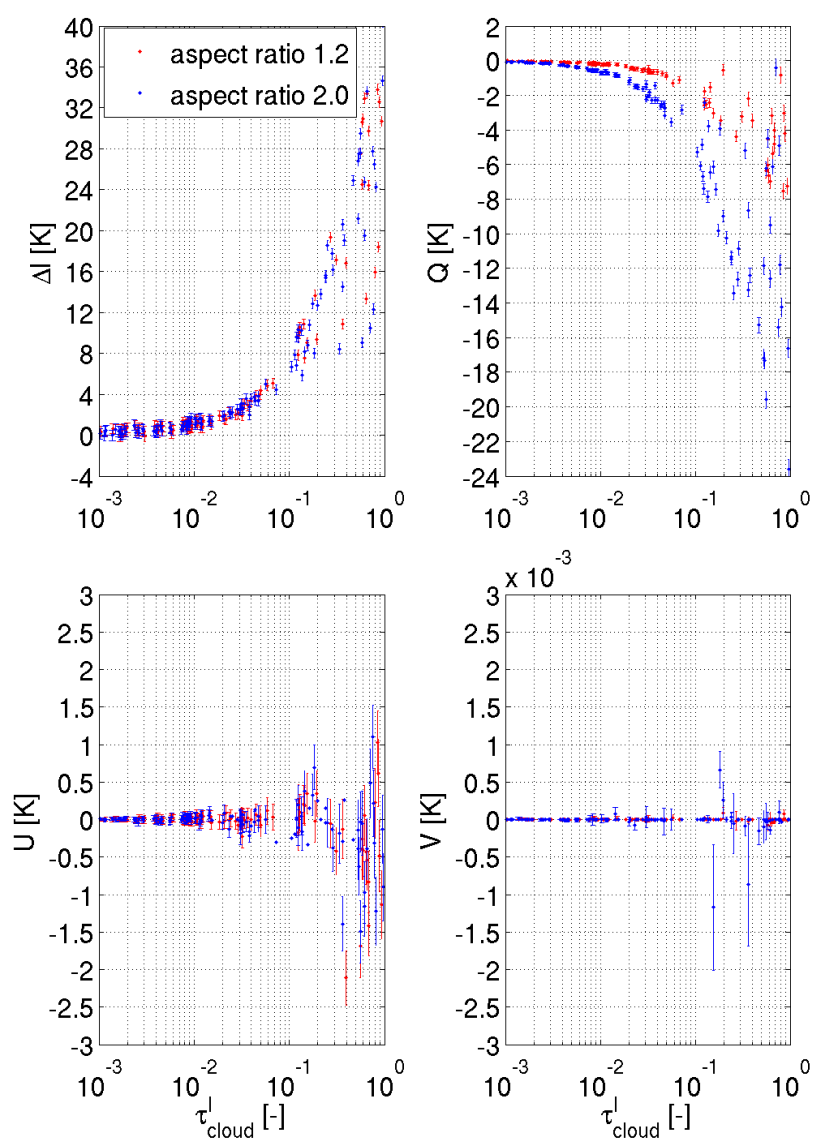

Fig. 5. Simulations of the cloud induced change of the observed Stokes vector for a collection of atmospheric states, as a function of cloud optical path. The simulations were performed for a frequency of $347.5 \mathrm{GHz}$ and a tangent altitude of $14 \mathrm{~km}$. The ice particles were modelled as horizontally aligned oblate spheroids with an aspect ratio of 1.2 (red) or 2.0 (blue). The vertical lines shows the error estimate $( \pm 2 \sigma)$ of the Monte Carlo radiative transfer method.

plot symbols. The figure shows that clouds embedded in an atmosphere with higher gaseous absorption give lower $\Delta I$, which is also consistent with Sect. 3.4. Some cases even show the characteristics of the "low" case with negative $\Delta I$.

High negative $\Delta I$ and negative $\Delta I$ for low clear-sky optical path $\tau_{\text {clear }}^{I}$ are indications of compact clouds, probably extending downwards and associated with deep convection. This situation can reduce the scattering source function to such a degree that $S<I$ for the $12 \mathrm{~km}$ tangent altitude. Clouds with the same optical path, but showing a high positive $\Delta I$ are likely widespread clouds with relatively low IWC.

\subsection{The scattering matrix}

The discussion of polarisation effects needs a basic understanding of the properties of the scattering matrix $(\mathbf{Z})$. For spherical particles and so called "macroscopically isotropic
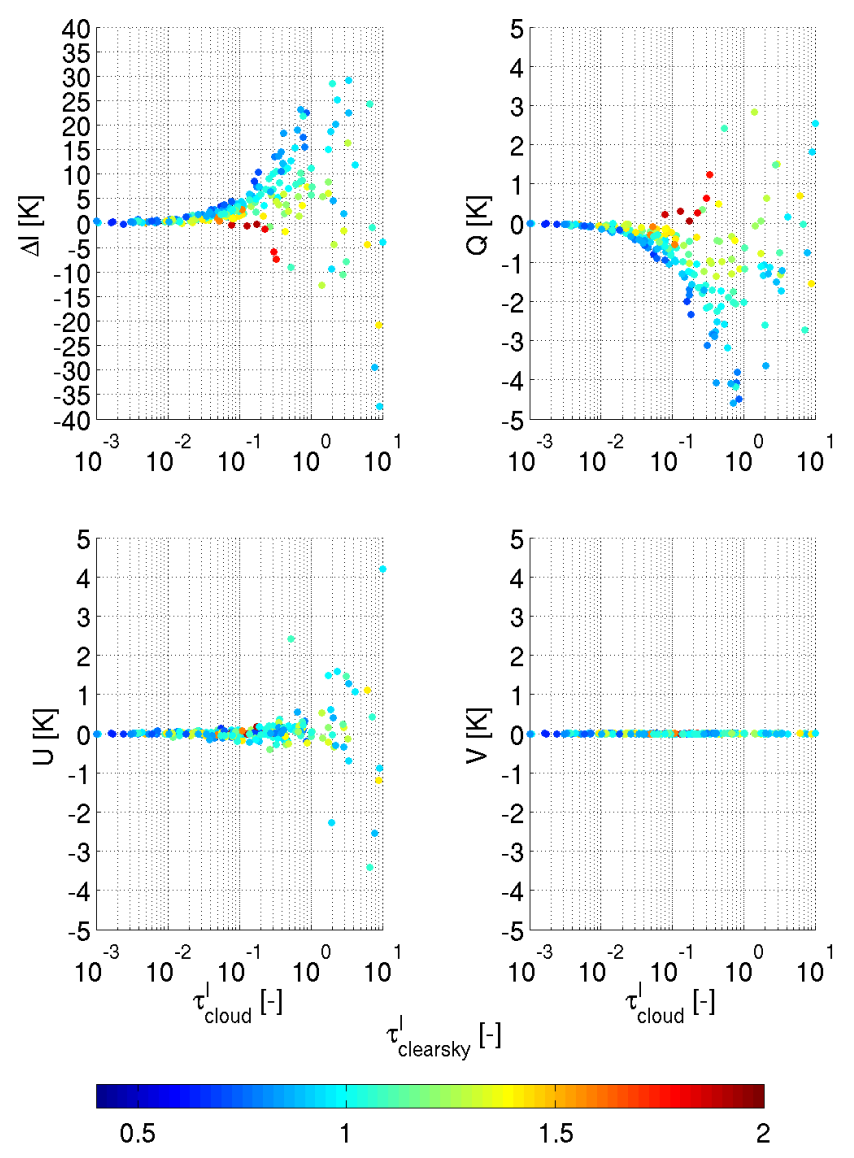

Fig. 6. Simulations of the cloud induced change of the observed Stokes vector for a collection of atmospheric states, as a function of cloud optical path. The simulations were performed for a frequency of $347.5 \mathrm{GHz}$ and a tangent altitude of $12 \mathrm{~km}$. The ice particles were modelled as horizontally aligned oblate spheroids with an aspect ratio of 1.2. The colours indicate the clear-sky optical path of the atmosphere (errors are of same size as in Fig. 5).

and symmetric media" they are well-described in textbooks (Mishchenko et al., 2002; Battaglia et al., 2006). The scattering matrix for horizontally aligned aspherical particles, as assumed here, is more complex.

Surface effects can be neglected for $347.5 \mathrm{GHz}$, and the radiation field inside the atmosphere without scattering is unpolarised $\left([I, 0,0,0]^{T}\right)$. If a single scatterer is placed in the atmosphere, the radiation scattered into the line-of-sight from each incoming direction is

$\left[Z_{11} I, Z_{21} I, Z_{31} I, Z_{41} I\right]^{T}$,

where $I$ is the incoming intensity and $Z_{i j}$ is the element of $\mathbf{Z}$ for row $i$ and column $j$. That is, for conditions of single scattering, only the first column of $\mathbf{Z}$ is of importance. Example values for this column are shown in Fig. 7.

The figure treats scattering from a zenith angle of $100^{\circ}$, to the limb-direction $\left(90^{\circ}\right)$. The absolute values of the azimuth angles do not matter, and the $\boldsymbol{n}$-direction is arbitrarily set to 
be at $0^{\circ}$. The exact backward and forward directions are not covered by the figure (they deviate with $10^{\circ}$ in the zenith direction), but they are relatively close compared to the width of the "scattering lobes" and the relative size of $Z_{11}$ at $0^{\circ}$ and $\pm 180^{\circ}$ shows that this particle exhibits somewhat stronger forward than backward scattering. This is expected since the particle has a size parameter of 0.62 , which is near the lower end of the Mie regime. (Rayleigh scattering would be equally strong in the forward and backward directions.)

\section{$4.2 Q$}

The magnitude of $Q$ is strongly influenced by particle shape and orientation (Czekala, 1998). For a given shape, the value of $Q$ is the smallest for randomly oriented particles. A comparison of Fig. 14 and 15 in Emde et al. (2004a) indicates that this orientation also has the most complex variation regarding the sign of $Q$.

For oriented particles, several studies have shown that the size of $Q$ depends on the asphericity (e.g. Miao et al., 2003), and this can also be seen in Fig. 5. With orientation, the signs of $\Delta I$ and $Q$ appear to be highly correlated (see Fig. 6, Fig. 15 of Emde et al. (2004a), and Fig. 2 of Davis et al., 2005a). To the degree orientation exists, it is expected that the longest dimension is found close to the horizontal plane (e.g. Bréon and Dubrulle, 2004), as also assumed in the setup of these simulations. In this case, $\Delta I$ and $Q$ vary in an anti-correlated manner. For "low" tangent altitudes $\Delta I$ is negative and $Q$ positive, and the opposite is valid for "high" altitudes.

More in detail, horizontally aligned oblate spheroids with an aspect ratio above one can be thought of having a greater size parameter in the horizontal direction than vertically (except for zenith angles of $0^{\circ}$ and $180^{\circ}$ where they are equal). This means that the extinction cross-section will be greater for the horizontally polarised radiation component than for the vertically polarised one. Consequently, for "low" tangent altitudes $Q$ is expected to be positive, as extinction dominates the cloud induced signal (Sect. 3.4). For "high" tangent altitudes $\Delta I$ and (thus also $Q$ ) is dominated by the contribution from radiation scattered into the line-of-sight. Since the particles have a greater horizontal than vertical scattering cross-section, more horizontal than vertical polarised radiation is scattered into the line-of-sight. Hence, we expect $Q$ to be negative for 'high' tangent altitudes.

However, the correlation between $\Delta I$ and $Q$ decreases with the strength of the scattering. This is most easily observed in Fig. 6, where the cases with highest $\tau$ are mainly found on the negative side for $\Delta I$ while $Q$ is centred around zero (indicating a correlation close to zero).

\section{$4.3 U$}

As mentioned in the introduction, we have not found any study on $U$ and $V$ for microwave limb sounding. An ex-

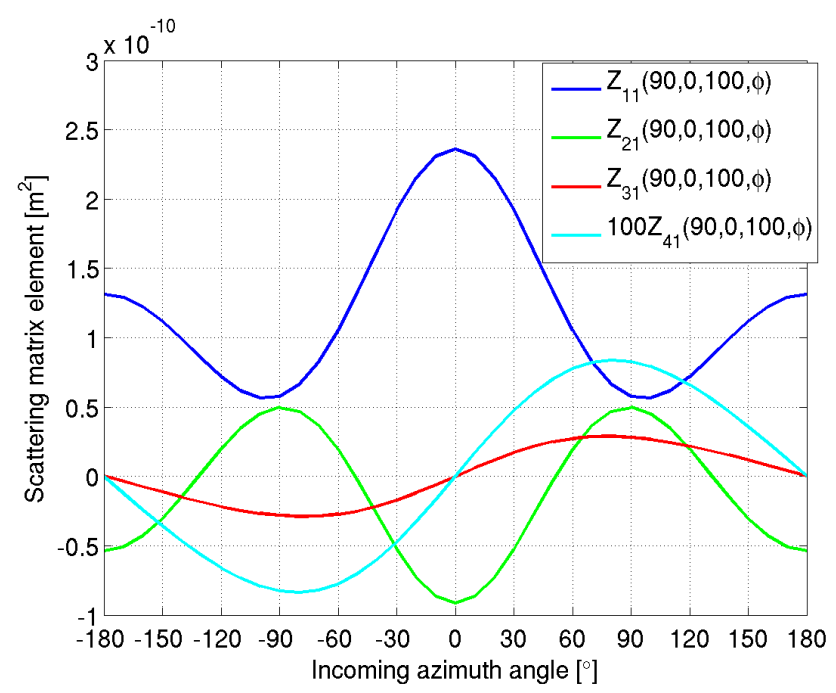

Fig. 7. Scattering matrix elements as a function of incoming azimuth angle, for horizontally aligned oblate spheroids with an aspect ratio of 2 , an equivalent diameter size of $170 \mu \mathrm{m}$ and a frequency of $347.5 \mathrm{GHz}$. In the nomenclature of Eq. (1), the zenith and azimuth angles of $\boldsymbol{n}$ are $90^{\circ}$ and $0^{\circ}$, respectively, and the zenith angle of $\boldsymbol{n}^{\prime}$ is $100^{\circ}$. The $Z_{41}$ is increased by a factor of 100 , to make the angular variation visible.

ample where $U$ is investigated for down-looking geometry is Adams et al. (2008).

In Figs. 5 and 6, $U$ deviates from zero only marginally for cloud optical path below 0.1 , i.e. weak cloud scattering. For these cases Eq. (14) gives a good description of the radiation scattered into the line-of-sight, where the term of concern here is $Z_{31} I$. Figure 7 shows that $Z_{31}$ is an odd function of incoming azimuth angle. The odd azimuthal symmetry of $Z_{31}$ can be understood from basic symmetry arguments and thus should be generally valid.

The radiation intensity field $I$, on the other hand, is expected to depend only weakly on azimuth angle. In combination with the odd symmetry of $Z_{31}$ this results in that the azimuthal integral of $Z_{31} I$, for each zenith angle, is close to zero. Hence, the contributions to $U$ from the 'left' and 'right' hemispheres for incoming radiation are more or less equally large, but have different sign, and the end result is close to zero.

For any given particle size, $Z_{31}$ depends only weakly on the incoming zenith angle (not shown). On the other hand, $Z_{31}$ varies strongly with particle size, where even the sign can change (Fig. 8). This means that small and large particles give counteracting contributions to $U$.

The azimuthal independence of $I$ is violated if the scattering point is surrounded by (inhomogeneous) clouds. The contributions from the left and right sides can then deviate significantly and the observed Stokes vector can have a significant $U$-value. In addition, the incoming radiation has then also a non-zero $Q$, which is converted to $U$ by elements of $\mathbf{Z}$ not covered by Eq. (14). 
In summary, a non-zero $U$ is primarily an effect of multiple scattering. This explains why $U$ has no preferred sign, as the cloud structures are random with respect to the observation direction. There is no systematic difference between $U$ for the two aspect ratios in Fig. 5. Some caution is needed when estimating the clear-sky influence on $U$. The atmospheric scenarios exhibit 3D structures for temperature and the gas constituents, but it is not clear to what extent horizontal variability is correctly modelled (Sect. 2.3).

\section{$4.4 \quad V$}

For these simulation conditions $V$ does not reach significant levels. The maximum (absolute) value for $V$ is only $0.035 \mathrm{~K}$, about three orders of magnitude below $U$. The $V$-element is a fairly parallel case to $U$, e.g. $Z_{41}$ and $Z_{31}$ show the same odd symmetry in Fig. 7 . The main difference is that $Z_{41} \ll Z_{31}$ for most particles. However, this depends on particle size, as shown in Fig. 8. The figure also shows that the switch from positive to negative values happens at a smaller size for $Z_{41}$ compared to $Z_{31}$ (around 400 and $600 \mu \mathrm{m}$, respectively). This should result in a higher cancellation between contributions from small and large particles for $V$ than for $U$.

\section{Retrieval aspects}

The implications of the results above for retrievals are discussed here. It is assumed that gaseous constituents are the main target of the measurements. The data in this section are reported as brightness temperatures for single polarisations, to match the response of real receivers, e.g.:

$T_{B}^{v}=I+Q / 2$,

which follows directly from Eqs. (2) and (3). The difference to the corresponding clear-sky case is denoted as $\Delta T_{b}$.

As noted above, inversions involving clouds are still a topic of research, and no actual retrievals are discussed here. Instead, some basic assumptions are made. It is assumed that clouds can be handled, at least, for single scattering situations. Figures 5 and 6 indicate that the break-point between single and multiple scattering is found near a cloud optical path of 0.1. This statement is based on the observation that $\Delta I$ shows low spread and $U$ is practically zero for $\tau^{I}<0.1$. It is further assumed that the measurements cannot provide any constraint for the particle shape.

\subsection{Selection of polarisation}

One aim of our study was to investigate which polarisation is theoretically best for the observations. If clouds are neglected in the retrievals, the polarisation should be selected such that their impact on the spectra is minimised. Considering the anti-correlation between $\Delta I$ and $Q$ (and that $Q$ is larger than $U$ and $V$ ), it is the vertical polarisation that fulfils

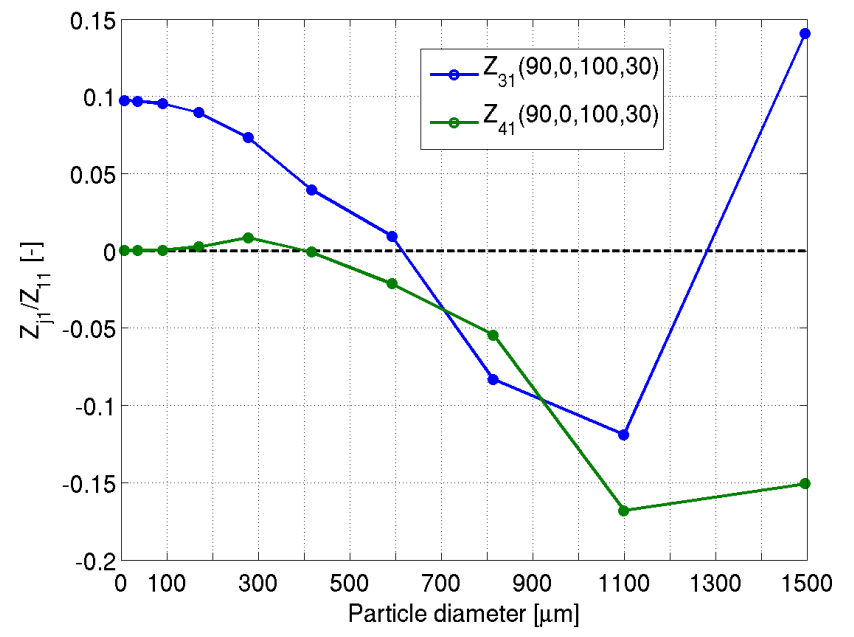

Fig. 8. Normalised scattering matrix elements as a function of particle equivalent diameter for horizontally aligned oblate spheroids with an aspect ratio of 2 , and a frequency of $347.5 \mathrm{GHz}$. In the nomenclature of Eq. 1, the zenith and azimuth angles of $\boldsymbol{n}$ are $90^{\circ}$ and $0^{\circ}$, respectively, and the zenith and azimuth angles of $\boldsymbol{n}^{\prime}$ are $100^{\circ}$ and $30^{\circ}$, respectively.

this criterion. As explained below, selecting lowest $\Delta T_{b}$, for a given cloud scenario, results in that the cloud extinction is also minimised.

A more elaborated approach is to include clouds in the retrieval process. The main consideration, with respect to the accuracy for gas species, is to obtain the best possible estimate of the extinction caused by clouds, in lack of a perfect knowledge on particle shapes. This translates into a demand of a compact relationship between cloud extinction and $\Delta T_{b}$, over a range of particle aspect ratios. (Size uncertainties are treated in Sect. 5.2.)

Some care is needed in this analysis. The "intensity" optical path used in Fig. 5 and $6\left(\tau^{I}\right)$ should not be used; $\Delta T_{b}$ is better compared to the optical path of the clouds for the polarisation of concern. That is, the extinction of interest is the one acting on the part of the gas emission having the measured polarisation. This quantity is here denoted as $\tau^{Z}$ and is calculated as $\tau^{I}$, but propagating polarised unit vectors through the atmosphere. For example, for vertical polarisation the initial Stokes vector is $[1,1,0,0]^{T}$ (instead of $[1,0,0,0]^{T}$ when calculating $\left.\tau^{I}\right)$.

As shown in Fig. 9, there is an equally compact relationship to $\Delta T_{b}$ for all polarisations and aspect ratios, as long as $\tau^{Z}$ is below $\approx 0.1$. Fits to the data in the lower panel of the figure give basically identical results for all cases. For small particles this is not a surprising finding. They are interacting with the radiation through absorption and emission, which are linked phenomena for LTE conditions.

For larger particles that cause scattering, Eq. (12) shows that $\Delta T_{b}$ is also proportional to $s$ (the scattering extinction) as long as the term $S$ is unchanged with particle shape. This 

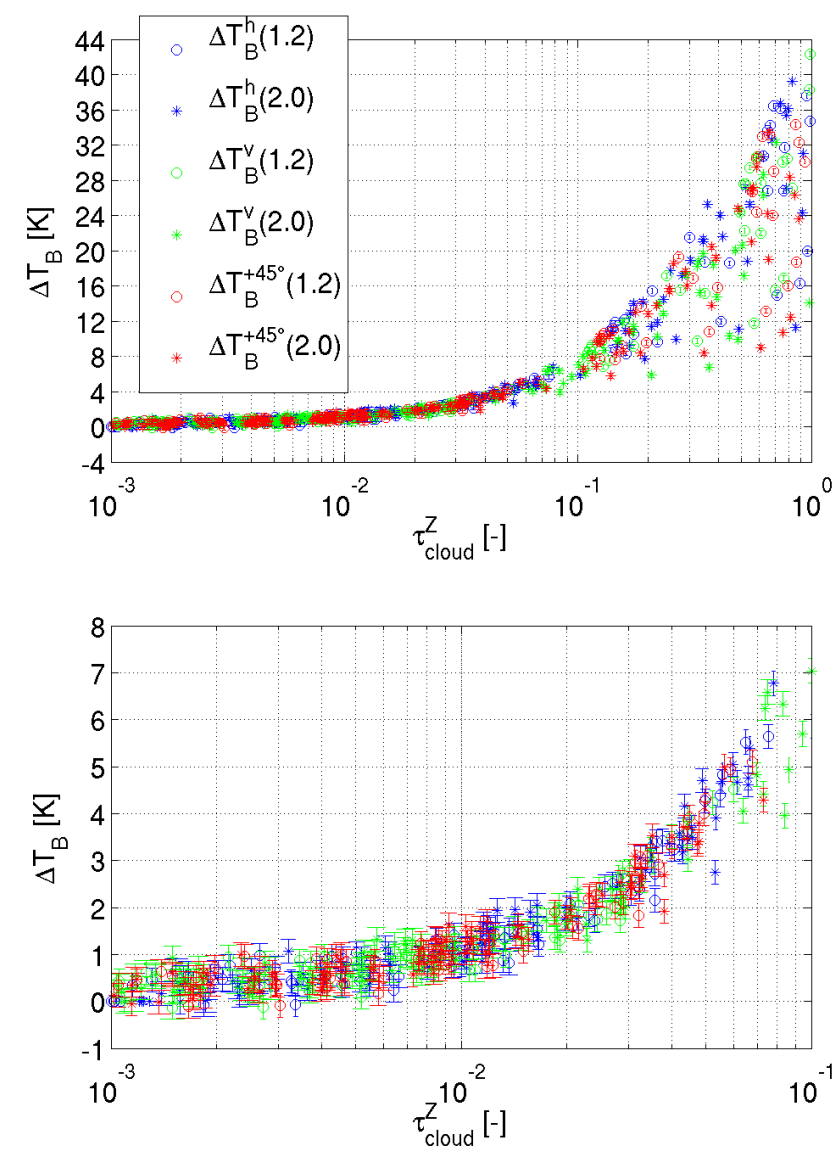

Fig. 9. Simulations of cloud induced signal, as function of polarisation specific cloud optical path, $\tau^{Z}$. The lower panel is a magnification of a part of the upper panel. The simulations are performed for a frequency of $347.5 \mathrm{GHz}$ and a tangent altitude of $14 \mathrm{~km}$.

is the case for Rayleigh sized particles, as the scattering function of Eq. (11) is not affected by particle shape (nor size) as long as $x \ll 1$. This is not valid for all particles, but, for the limb direction, deviations from the Rayleigh scattering function have only a relatively small influence. The reason for this is that the radiation scattered into the line-of-sight can be approximated as step function (see Fig. 3 of Emde et al., 2004a), the up-welling and down-welling parts are each more or less constant with zenith angle (but have different $T_{b}$ ). For limb observations, a change of the scattering function is close to symmetric around the break-point of the step function, and the net effect on $S$ becomes small. This is a simplified treatment of the scattering source term, but the results of Fig. 9 show that it is valid in practice. Another way to express this is that the asymmetry factor (in short, the ratio between forward and backward scattering) is of smaller interest for these measurements. But note that this symmetry applies only to the limb direction.

The compact relationship to $\Delta T_{b}$ is not found for higher cloud $\tau^{Z}$, which is a result of multiple scattering. No sys-

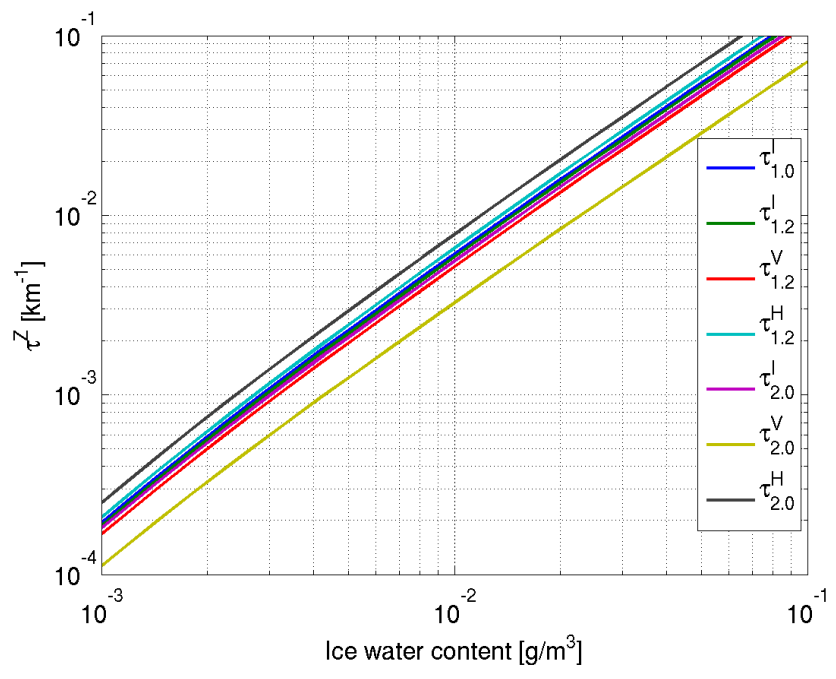

Fig. 10. Cloud ice optical paths for a $90^{\circ}$ zenith angle and horizontally aligned oblate spheroids with an aspect ratio of 1, 1.2 and 2.0. Calculated with the PSD of MH97 and a temperature of $220 \mathrm{~K}$. The different lines show the optical path for different aspect ratios and polarisation components (where $I$ represents several components, as explained in text).

tematic pattern between the polarisations can be discerned. The conclusion is that all polarisations are equally good with respect to gas species retrievals, and that it should be possible to maintain an acceptable accuracy up to cloud optical path around 0.1 .

However, the possibility of also retrieving cloud properties should not be forgotten. The main aim for observations of this type is to estimate the IWC, and the desired property for these retrievals is smallest possible impact of particle shape on the relationship between particle extinction and IWC.

Figure 10 shows that the vertical and horizontal polarisations lack this feature. This follows from the discussion in Sect. 4.2, explaining that the effective cross-section differs between $V$ and $H$ polarisation, and this difference increases with the aspect ratio. As an example, if $V$ polarisation is measured, it is not clear if the blue, red or yellow curve should be used to map cloud extinction to IWC, assuming that the particle asphericity can not be determined by some other means.

This can also be explained by examining the extinction matrix, which has the structure

$\mathbf{K}(\theta)=\left[\begin{array}{cccc}K_{11}(\theta) & K_{12}(\theta) & 0 & 0 \\ K_{12}(\theta) & K_{11}(\theta) & 0 & 0 \\ 0 & 0 & K_{11}(\theta) & K_{34}(\theta) \\ 0 & 0 & -K_{34}(\theta) & K_{11}(\theta)\end{array}\right]$,

where $\theta$ is the zenith angle. Thus, the (scalar) extinction coefficient for the vertical component $\left([1,1,0,0]^{T}\right)$ is $K_{11}+$ $K_{12}$, while for the horizontal one $\left([1,-1,0,0]^{T}\right)$ it is $K_{11}-$ $K_{12}$. That is, the extinction for $\mathrm{V}$ and $\mathrm{H}$ differs. 
Using the same reasoning, Eq. (16) shows also that the extinction coefficient is the same for the $\pm 45^{\circ}$ linear and left/right-hand circular components, and it is $K_{11}$. This is also the extinction coefficient for total intensity, and $I$ is therefore used as the label in Fig. 10 for the common cloud optical path associated with $T_{B}^{+45^{\circ}}, T_{B}^{-45^{\circ}}, T_{B}^{\text {lhc }}$ and $T_{B}^{\text {rhc }}$. But keep in mind that $I$ itself usually can not be measured directly.

It turns out that the $K_{11}$-element is only weakly affected by the aspect ratio (maintaining a constant volume) for the influential particle sizes, and the curves in Fig. 10 of $\tau^{I}$ for different aspect ratios are close to identical. Hence, the mapping from extinction to IWC for observations of $T_{B}^{+45^{\circ}}, T_{B}^{-45^{\circ}}$, $T_{B}^{\text {lhc }}$ and $T_{B}^{\text {rhc }}$ has a low sensitivity to uncertainties regarding the particle asphericity, which is an advantage over the $\mathrm{V}$ and H options.

Spheroidal particles were assumed in this study, and the situation is more complex for more realistic particle shapes. However, the discussion above should be relevant as long as the particles have random azimuthal orientation and no particular shape symmetry.

\subsection{Representation of particle sizes}

Conceptually, the simplest option for the retrievals is to assume a PSD parametrisation, exactly as done for the simulations here. A general parametrisation, such as MH97, could potentially describe average conditions correctly, but the instantaneous local PSD can deviate strongly. That is, a general relationship between IWC and extinction does not exist. Accordingly, IWC is not a suitable variable to represent the cloud properties and it should instead be better to directly retrieve the relevant optical properties of the cloud particles.

Each particle type that is introduced into the representation of the optical properties causes an additional calculation cost. Furthermore, the observations provide even in the best case just coarse information on the PSD. Thus, the number of variables to describe the particles should be kept as low as possible. But what is the minimum number of variables required to represent the particle properties?

In Sect. 3 it was shown that particle absorption can not be neglected, and that the source functions associated with emission and scattering differ. Hence, at least two variables are needed to give the retrieval a possibility of fitting effects originating from both absorption and scattering. These effects can in rough terms be assigned to 'small' and 'large' particles, respectively.

Absorption effects are fully described by the absorption coefficient $(a)$, and a single size is sufficient to represent the impact of the small particles. If this absorption coefficient can be determined, it can be translated to an IWC with high accuracy, as absorption in the Rayleigh domain is proportional to the particle volume (Sect. 3.2).

The results in Sect. 5.1 indicate that an exact representation of the scattering function is not needed, since the rela- tionship between cloud scattering extinction and $\Delta T_{b}$ is only weakly affected by the particle size. Consequently, the effects of scattering can also fairly well be represented by a single particle size. A particle size that gives a single scattering albedo close to 1 should be selected if it is important to maintain a clear distinction to the absorption of the small mode. However, it should be more important to maintain a high similarity between the scattering functions of the particle type applied and the true particle ensemble. This points towards using a particle size around $200 \mu \mathrm{m}$, as the product of scattering cross-section and the PSD (assuming MH97) peaks in the range 150-300 $\mu \mathrm{m}$ (Wu et al., 2005; Eriksson et al., 2008).

A representation in terms of different particle sizes is assumed above. The task of the retrieval is then to determine the number density of each particle size. A similar option is to instead operate directly with the optical properties. In terms of Eq. (8), the two retrieval quantities are then the absorption $(a)$ and scattering coefficients $(s)$. The normalised scattering function $(p)$ can either be fixed or given a predefined relation to $s$.

The discussion above assumes that the retrieval can estimate absorption and scattering, at least partly, as independent effects, but to what extent this is possible in practical retrievals is not investigated here. However, the possibility of separating the two effects increases if data from two or more wavelength bands can be combined. For single band retrievals, a high variation of the gaseous absorption over the band should be required. If the effects can not be separated, the clouds have to be covered by a single retrieval variable, with a pre-defined relationship between absorption and scattering, most likely following a PSD parametrisation.

\section{Conclusions}

We performed detailed simulations of microwave limb sounding at $347.5 \mathrm{GHz}$ involving ice cloud scattering. The input to the simulations was generated with care, including three dimensional cloud scenarios, to achieve as realistic simulations as possible. The primary aim of the simulations was to improve the general understanding of absorption and polarisation effects caused by ice clouds. Hence, the purpose was not to provide exact statistics of cloud induced radiance changes. This is anyhow not possible as some input variables have large uncertainties, where particle shape and sizes are the most prominent examples.

A main conclusion is that the impact of particle absorption is of much higher concern for limb sounding than for down-looking measurements. This difference results from a combination of two factors. Firstly, limb sounding gives emphasis to higher altitudes where the ice particles tend to have smaller sizes. This gives a higher relative importance of absorption for the particle extinction. Secondly, there is a higher contrast between the "radiative background" and the 
emission source term for limb sounding. For down-looking data this contrast is close to zero, any particle absorption is replaced by particle emission of almost the same magnitude and the net effect is small. These aspects are not covered by the single scattering albedo, the standard measure on the ratio between scattering and total extinction.

This conclusion has several practical consequences. A first consideration is the generation of the atmospheric scenarios. The magnitude of scattering at $347.5 \mathrm{GHz}$ is constrained fairly well by the CloudSat data from $94 \mathrm{GHz}$. Rayleigh conditions in general apply for both frequencies and the strength of scattering can be scaled as $\lambda_{1}^{4} / \lambda_{2}^{4}$. On the other hand, CloudSat gives no information on particle absorption and the magnitude of absorption thus becomes a consequence of the assumed particle size distribution (PSD). However, PSD parameterisations must be judged as uncertain and the application of a single PSD for all cloud types is a strong simplification. This generates a substantial uncertainty for some of the results, such as the exact values for $\Delta I$ and relative importance of absorption.

Furthermore, no general relationship between cloud extinction and $\Delta T_{b}$ can be established, it depends on the ratio between particle absorption and scattering. This forces the retrieval to handle the cloud optical properties with at least two variables, and, if the measurements provide the necessary information, retrieve these variables as independent quantities. This in order to both avoid systematic errors for the gas constituents and to achieve a correct mapping of the cloud extinction to ice water content.

These simulations confirm earlier results regarding the $Q$ element of the Stokes vector. The difference between the brightness temperature of the vertical $\left(T_{B}^{v}\right)$ and horizontal $\left(T_{B}^{h}\right)$ linear components increases with the aspect ratio of the particles, assuming that they have a tendency for orientation alignment. An azimuthal orientation of the particles would give an impact of particle shape also on the higher Stokes elements, $U$ and $V$, but this effect has not been studied due to the lack of useful input for the simulations. For oblate spheroids, or particles with random azimuthal orientation, $U$ and $V$ are instead controlled by azimuthal inhomogeneities in the radiation field. These simulations generate radiances that are more or less constant with the azimuthal angle in the absence of clouds, and such inhomogeneities are mainly associated with cloud effects already in the radiation to be scattered into the line-of-sight, i.e. multiple scattering. The value of $U$ can be significant, while the maximum value of $V$ obtained here is below $0.035 \mathrm{~K}$. This is due to the lower values of the relevant scattering matrix element for $V$, and also a higher cancellation of contributions from particles of different size.

The results indicate that single scattering can be assumed for cloud optical paths $(\tau)$ below about 0.1 . For example, nonzero values for $U$ and $V$ are only found above that level. In addition, it is shown that for these conditions of thinner clouds there is a compact relationship between $\tau$ and $\Delta T_{b}$, independent of particle shape and observed polarisation component measured. This in its turn signifies that it should be possible to perform gas species retrievals with high accuracy for $\tau<0.1$.

With respect to the retrieval of gas constituents, there is thus only a small difference between the polarisation options. Measuring the vertical component has a slight advantage since it minimises the cloud extinction. On the other hand, we showed that observing $T_{B}^{v}$ or $T_{B}^{h}$ leads to a dependence on the particle aspect ratio for the conversion from cloud extinction to ice water content (IWC). As $U$ and $V$ have small values (at least for low $\tau$ ), using any of the $\pm 45^{\circ}$ or circular components is basically identical to measuring the total intensity. The intensity equals the average of the $T_{B}^{v}$ and $T_{B}^{h}$ components, and the brightness temperature of this average is much less affected by the particle shape than $T_{B}^{v}$ and $T_{B}^{h}$ separately. Hence, for retrievals of IWC, it is preferable to select a polarisation component associated with $U$ or $V$.

A related aspect is to make use of measurements of the same air volume from two orthogonal polarisations. If both measurements are from an identical position, no additional information is obtained by observing the $\pm 45^{\circ}$ or circular components, while the combination of the $T_{B}^{v}$ and $T_{B}^{h}$ components gives a means for a simple detection of cloud influences and possibly also an estimation of the particle aspect ratio. However, if the two measurements come from two different positions, it is hard to disentangle to what extent differences in $\Delta T_{b}$ are generated by particle shape effects and changed propagation path through the cloud. In the later case, it should be better to avoid the influence of particle shape by using either the $\pm 45^{\circ}$ or circular components, and focus on using the two measurements to improve the spatial resolution of the retrieval. This discussion is directly applicable to the PREMIER mm limb sounder, and this study confirms the initial decision to use $\pm 45^{\circ}$ polarisation for the overlapping beams of this instrument.

Despite the fact that the simulations can be considered as quite detailed, they have several limitations that must be noted. Complete radiative transfer was only performed for a single frequency. For example, if a higher frequency would have been selected, a smaller relative importance of absorption would have been found. The main microphysical variable governing the degree of polarisation should be the (bulk) aspect ratio, and here two different values were used. However, it should be kept in mind that a single particle size distribution was applied and no other particle shape than spheroids was considered. As long as the particles can be assumed to have no preferred azimuthal orientation, this simplification should be acceptable, but highly deviating results could be obtained by assuming oriented particles of special shapes, especially for Stokes elements $U$ and $V$. 
Acknowledgements. We thank the ARTS community, and particularly Cory Davis who implemented the Monte Carlo module that we have used for the radiative transfer simulations. The publicly available CloudSat data were an important part of the study. The work was performed as part of an ESA funded project: PREMIER, Consolidation of Requirements and Synergetic Retrieval Algorithms ("CORSA", ESTEC Contract No. 22848/09/NL/CT). Stefan Buehler's contribution was funded by the Swedish Space Board, under the research program "Satellite Atmospheric Science".

Edited by: A. Lambert

\section{References}

Adams, I. S., Gaiser, P., and Jones, W. L.: Simulation of the Stokes vector in inhomogeneous precipitation, Radio Sci., 43, RS5006, doi:10.1029/2007RS003744, 2008.

Battaglia, A., Simmer, C., Crewell, S., Czekala, H., Emde, C., Marzano, F., Mishchenko, M., Pardo, J., and Prigent, C.: Emission and scattering by clouds and precipitation, in: Thermal microwave radiation: Applications for remote sensing, edited by: Mätzler, C., 101-233, The Institution of Engineering and Technology, London, UK, 2006.

Bohren, C. F. and Huffman, D. R.: Absorption and scattering of light by small particles, Wiley, New York, USA, 1998.

Bréon, F. and Dubrulle, B.: Horizontally oriented plates in clouds, J. Atmos. Sci., 61, 2888-2898, 2004.

Buehler, S. A., Eriksson, P., Kuhn, T., von Engeln, A., and Verdes, C.: ARTS, the Atmospheric Radiative Transfer Simulator, J. Quant. Spectrosc. Radiat. Transfer, 91, 65-93, 2005.

Buehler, S. A., Jiménez, C., Evans, K. F., Eriksson, P., Rydberg, B., Heymsfield, A. J., Stubenrauch, C., Lohmann, U., Emde, C., John, V. O., Sreerekha, T. R., and Davis, C. P.: A concept for a satellite mission to measure cloud ice water path and ice particle size, Q. J. R. Meteorol. Soc., 133, 109-128, 2007.

Buehler, S. A., Eriksson, P., and Lemke, O.: Absorption lookup tables in the radiative transfer model ARTS, J. Quant. Spectrosc. Radiat. Transfer, 112, 1559-1567, doi:10.1016/j.jqst.2011.03.008, 2011.

Chandrasekhar, S.: Radiative transfer, Dover, New York, 1950.

Czekala, H.: Effects of particle shape and orientation on polarized microwave radiation for off-nadir problems, Geophys. Res. Lett., 25, 1669-1672, 1998.

Davis, C., Emde, C., and Harwood, R.: A 3D polarized reversed Monte Carlo radiative transfer model for $\mathrm{mm}$ and sub-mm passive remote sensing in cloudy atmospheres, IEEE Trans. Geosci. Remote Sensing, 43, 1096-1101, 2005a.

Davis, C. P., Wu, D. L., Emde, C., Jiang, J. H., Cofield, R. E., and Harwood, R. S.: Cirrus induced polarization in $122 \mathrm{GHz}$ Aura Microwave Limb Sounder radiances, Geophys. Res. Lett., 32, L14806, doi:10.1029/2005GL022681, 2005b.

Davis, C. P., Evans, K. F., Buehler, S. A., Wu, D. L., and Pumphrey, H. C.: 3-D polarised simulations of space-borne passive $\mathrm{mm} / \mathrm{sub}-$ mm midlatitude cirrus observations: a case study, Atmos. Chem. Phys., 7, 4149-4158, doi:10.5194/acp-7-4149-2007, 2007.

Ekström, M., Eriksson, P., Rydberg, B., and Murtagh, D. P.: First Odin sub-mm retrievals in the tropical upper troposphere: humidity and cloud ice signals, Atmos. Chem. Phys., 7, 459-469, doi:10.5194/acp-7-459-2007, 2007.
Ekström, M., Eriksson, P., Read, W. G., Milz, M., and Murtagh, D. P.: Comparison of satellite limb-sounding humidity climatologies of the uppermost tropical troposphere, Atmos. Chem. Phys., 8, 309-320, doi:10.5194/acp-8-309-2008, 2008.

Emde, C., Buehler, S. A., Davis, C., Eriksson, P., Sreerekha, T. R., and Teichmann, C.: A polarized discrete ordinate scattering model for simulations of limb and nadir longwave measurements in 1D/3D spherical atmospheres, J. Geophys. Res., 109(D24), D24207, doi:10.1029/2004JD005140, 2004a.

Emde, C., Buehler, S. A., Eriksson, P., and Sreerekha, T. R.: The effect of cirrus clouds on limb radiances, J. Atmos. Res., 72, 383401, 2004b.

Eriksson, P., Ekström, M., Rydberg, B., and Murtagh, D. P.: First Odin sub-mm retrievals in the tropical upper troposphere: ice cloud properties, Atmos. Chem. Phys., 7, 471-483, doi:10.5194/acp-7-471-2007, 2007.

Eriksson, P., Ekström, M., Rydberg, B., Wu, D. L., Austin, R. T., and Murtagh, D. P.: Comparison between early Odin-SMR, Aura MLS and CloudSat retrievals of cloud ice mass in the upper tropical troposphere, Atmos. Chem. Phys., 8, 1937-1948, doi:10.5194/acp-8-1937-2008, 2008.

Eriksson, P., Rydberg, B., Johnston, M., Murtagh, D. P., Struthers, H., Ferrachat, S., and Lohmann, U.: Diurnal variations of humidity and ice water content in the tropical upper troposphere, Atmos. Chem. Phys., 10, 11519-11533, doi:10.5194/acp-1011519-2010, 2010.

Eriksson, P., Buehler, S. A., Davis, C. P., Emde, C., and Lemke, O.: ARTS, the atmospheric radiative transfer simulator, Version 2, J. Quant. Spectrosc. Radiat. Transfer, 112, 1551-1558, doi:10.1016/j.jqsrt.2011.03.001, 2011.

ESA: PREMIER, report for assessment, Tech. rep., European Space Agency, http://esamultimedia.esa.int/docs/ SP1313-5_PREMIER.pdf, accessed: 1 March 2011, 2008.

Evans, K. F. and Stephens, G. L.: Microwave radiative transfer through clouds composed of realistically shaped ice crystals. Part I. Single scattering properties, J. Atmos. Sci., 52, 2041-2057, 1995a.

Evans, K. F. and Stephens, G. L.: Microwave radiative transfer through clouds composed of realistically shaped ice crystals. Part II. Remote sensing of ice clouds, J. Atmos. Sci., 52, 2058-2072, 1995b.

Evans, K. F., Walter, S. J., Heymsfield, A. J., and McFarquhar, G. M.: Submillimeter-wave cloud ice radiometer: Simulations of retrieval algorithm performance, J. Geophys. Res., 107, 2.12.21, 2002.

Kikuchi, K., Nishibori, T., Ochiai, S., Ozeki, H., Irimajiri, Y., Kasai, Y., Koike, M., Manabe, T., Mizukoshi, K., Murayama, Y., Nagahama, T., Sano, T., Sato, R., Seta, M., Takahashi, C., Takayanagi, M., Masuko, H., Inatani, J., Suzuki, M., and Shiotani, M.: Overview and early results of the Superconducting Submillimeter-Wave Limb-Emission Sounder (SMILES), J. Geophys. Res., 115, D23306, doi:10.1029/2010JD014379, 2010.

Li, J.-L., Waliser, D. E., Jiang, J., Wu, D. L., Read, W., Waters, J. W., Tompkins, A. M., Donner, L. J., Chern, J.-D., Tao, W.-K., Atlas, R., Gu, Y., Liou, K. N., Genio, A. D., Khairoutdinov, M., and Gettelman, A.: Comparisons of EOS MLS cloud ice measurements with ECMWF analyses and GCM simulations: Initial results, Geophys. Res. Lett., 32, L18710, doi:10.1029/2005GL023788, 2005. 
Liebe, H. J., Hufford, G. A., and Cotton, M. G.: Propagation modeling of moist air and suspended water/ice particles at frequencies below $1000 \mathrm{GHz}$, in: AGARD conference proceedings 542: Atmospheric propagation effects through natural and man-made obscurants for visible to mm-wave radiation, 3.1-3.10, Palma de Mallorca, Spain, 17-20 May 1993, 1993.

Livesey, N. J., Snyder, W. V., Read, W. G., and Wagner, P. A.: Retrieval algorithms for the EOS Microwave Limb Sounder (MLS), IEEE Trans. Geosci. Remote Sensing, 44, 1144-1155, 2006.

McFarquhar, G. M. and Heymsfield, A. J.: Parameterization of tropical cirrus ice crystal size distribution and implications for radiative transfer: Results from CEPEX, J. Atmos. Sci., 54, 21872200, 1997.

Miao, J., Johnsen, K.-P., Buehler, S., and Kokhanovsky, A.: The potential of polarization measurements from space at $\mathrm{mm}$ and sub$\mathrm{mm}$ wavelengths for determining cirrus cloud parameters, Atmos. Chem. Phys., 3, 39-48, doi:10.5194/acp-3-39-2003, 2003.

Mishchenko, M. I., Travis, L., and Lacis, A.: Scattering, absorption, and emission of light by small particles, Cambridge University Press, Cambridge, UK, 2002.

Murtagh, D., Frisk, U., Merino, F., Ridal, M., Jonsson, A., Stegman, J., Witt, G., Eriksson, P., Jiménez, C., Megie, G., de La Noë, J., Ricaud, P., Baron, P., Pardo, J. R., Hauchcorne, A., Llewellyn, E. J., Degenstein, D. A., Gattinger, R. L., Lloyd, N. D., Evans, W. F. J., McDade, I. C., Haley, C., Sioris, C., von Savigny, C., Solheim, B. H., McConnell, J. C., Strong, K., Richardson, E. H., Leppelmeier, G. W., Kyrölä, E., Auvinen, H., and Oikarinen, L.: An overview of the Odin atmospheric mission, Can. J. Phys., 80, 309-319, 2002.

Okamoto, H., Sato, K., and Hagihara, Y.: Global analysis of ice microphysics from CloudSat and CALIPSO: Incorporation of specular reflection in lidar signals, J. Geophys. Res., 115, D22209, doi:10.1029/2009JD013383, 2010.

Rosenkranz, P. W.: Absorption of microwaves by atmospheric gases, in: Atmospheric remote sensing by microwave radiometry, edited by: Janssen, M. A., 37-90, John Wiley and Sons, Inc., New York, USA, 1993.

Rosenkranz, P. W.: Water vapor microwave continuum absorption: A comparison of measurements and models, Radio Sci., 33, 919928, (correction in 34, 1025, 1999), 1998.

Rydberg, B., Eriksson, P., and Buehler, S. A.: Prediction of cloud ice signatures in submillimetre emission spectra by means of ground-based radar and in-situ microphysical data, Q. J. R. Meteorol. Soc., 133, 151-162, 2007.
Rydberg, B., Eriksson, P., Buehler, S. A., and Murtagh, D. P.: NonGaussian Bayesian retrieval of tropical upper tropospheric cloud ice and water vapour from Odin-SMR measurements, Atmos. Meas. Tech., 2, 621-637, doi:10.5194/amt-2-621-2009, 2009.

Stephens, G. L., Vane, D. G., Boain, R. J., Mace, G. G., Sassen, K., Wang, Z. E., Illingworth, A. J., O'Connor, E. J., Rossow, W. B., Durden, S. L., Miller, S., Austin, R. T., Benedetti, A., and Mitrescu, C.: The CloudSat mission and the A-train - A new dimension of space-based observations of clouds and precipitation, Bull. Amer. Met. Soc., 83, 1771-1790, 2002.

Teichmann, C., Buehler, S. A., and Emde, C.: Understanding the polarization signal of spherical particles for microwave limb radiances, J. Quant. Spectrosc. Radiat. Transfer, 101, 179-190, 2006.

Urban, J., Lautié, N., Le Flochmoën, E., Jiménez, C., Eriksson, P., Dupuy, E., El Amraoui, L., Ekström, M., Frisk, U., Murtagh, D., de La Noë, J., Olberg, M., and Ricaud, P.: Odin/SMR limb observations of stratospheric trace gases: level 2 Processing of $\mathrm{ClO}, \mathrm{N}_{2} \mathrm{O}, \mathrm{O}_{3}$, and $\mathrm{HNO}_{3}$, J. Geophys. Res., 110, D14307, doi:10.1029/2004JD005741, 2005.

Venema, V., Ament, F., and Simme, C.: A stochastic iterative amplitude adjusted Fourier transform algorithm with improved accuracy, Nonlin. Processes Geophys, 13, 321-328, 2006.

Warren, S. G. and Brandt, R. E.: Optical constants of ice from the ultraviolet to the microwave: A revised compilation, J. Geophys. Res., 113, D14220, doi:10.1029/2007JD009744, 2008.

Waters, J. W., Froidevaux, L., Harwood, R. S., Jarnot, R. F., Pickett, H. M., Read, W., Siegel, P. H., Cofield, R. E., Filipiak, M. J., Flower, D. A., Holden, J. R., Lau, G. K., Livesey, N. J., Manney, G. L., Pumphrey, H. C., Santee, M. L., Wu, W. L., Cuddy, D. T., Lay, R. R., Loo, M. S., Perun, V. S., Schwartz, M. J., Stek, P., Thurstans, R. P., Boyles, M. A., Chandra, K. M., Chavez, M. C., Chen, G. S., Chudasama, B. V., Dodge, R., Fuller, R. A., Girard, M. A., Jiang, J. H., Jiang, Y. B., Knosp, B. W., LaBelle, R., Lam, J. C., Lee, K. A., Miller, D., Oswald, J. E., Patel, N. C., Pukala, D. M., Quintero, O., Scaff, D. M., Snyder, W. V., Tope, M. C., Wagner, P., and Walch, M. J.: The Earth Observing System Microwave Limb Sounder (EOS MLS) on the Aura satellite, IEEE Trans. Geosci. Remote Sensing, 44, 1075-1092, 2006.

Wu, D. L., Read, W. G., Dessler, A. E., Sherwood, S. C., and Jiang, J. H.: UARS/MLS cloud ice measurements: Implications for $\mathrm{H}_{2} \mathrm{O}$ transport near the tropopause, J. Atmos. Sci., 62, 518-530, 2005.

Wu, D. L., Jiang, J. H., and Davis, C. P.: EOS MLS cloud ice measurements and cloudy-sky radiative transfer model, IEEE Trans. Geosci. Remote Sensing, 44, 1156-1165, 2006. 